\title{
Insulin Resistance Does Not Influence Gene Expression in Skeletal Muscle
}

\author{
Lisa L. Nguyen ${ }^{1, *}$, Adamandia D. Kriketos ${ }^{2}$, Dale P. Hancock ${ }^{1}$, Ian D. Caterson ${ }^{1}$ and Gareth S. Denyer ${ }^{1}$ \\ ${ }^{1}$ School of Molecular and Microbial Biosciences, University of Sydney, Sydney, NSW, Australia \\ ${ }^{2}$ Diabetes and Obesity Research Program, Garvan Institute of Medical Research, Sydney, NSW, Australia
}

Received 21 February 2006, Accepted 30 May 2006

\begin{abstract}
Insulin resistance is commonly observed in patients prior to the development of type 2 diabetes and may predict the onset of the disease. We tested the hypothesis that impairment in insulin stimulated glucose-disposal in insulin resistant patients would be reflected in the gene expression profile of skeletal muscle. We performed gene expression profiling on skeletal muscle of insulin resistant and insulin sensitive subjects using microarrays. Microarray analysis of 19,000 genes in skeletal muscle did not display a significant difference between insulin resistant and insulin sensitive muscle. This was confirmed with real-time PCR. Our results suggest that insulin resistance is not reflected by changes in the gene expression profile in skeletal muscle.
\end{abstract}

Keywords: Gene expression, Insulin resistance, Microarray, Real-time PCR, Skeletal muscle

\section{Introduction}

Insulin resistance is characterised by a reduced sensitivity to the actions of insulin. In response circulating insulin levels are increased in order to stimulate peripheral glucose disposal and maintain euglycaemia. This compensatory increase in insulin is usually observed before the detection of hyperglycaemia per se (Goldstein, 2002; Goldstein, 2003; Haffner, 2003a; Haffner, 2003b) . In most cases, type 2 diabetes results from the body's inability to maintain this compensatory hyperinsulinaemia (Yki-Jarvinen, 1995; Goldstein, 2002; Smith, 2002; Goldstein, 2003; Haffner, 2003a; Haffner, 2003b).

As well as being an important factor in NIDDM insulin resistance is an early risk factor of the metabolic syndrome

\footnotetext{
Abbreviations: NIDDM non-insulin dependent diabetes mellitus, GIR glucose infusion rate, FFM fat free mass
}

\footnotetext{
*To whom correspondence should be addressed.

Tel: 61-2-93516024; Fax: 61-2-93514726

E-mail: L.Nguyen@mmb.usyd.edu.au
}

and is associated with diseases such as hypertension, obesity and cardiovascular disease (Kahn and Flier, 2000; Kopelman, 2000; Felber and Golay, 2002; Goldstein, 2003; Haffner, 2003b; Dandona et al., 2004; Sowers, 2004).

Skeletal muscle is responsible for the majority of insulin stimulated glucose disposal. A decline in the body's responsiveness to insulin most probably results from a defect in this tissue. It has been shown that lean, normoglycaemic offspring of parents with NIDDM have a 70\% reduction in muscle glycogen synthesis and a $40 \%$ reduction in Glucose-6Phosphate concentration (Petersen and Shulman, 2002).

However, it is known that whole body insulin resistance does not necessarily arise from skeletal muscle alone and that liver and adipose tissue could be major contributors to this whole body insulin resistance.

The exact molecular mechanism by which skeletal muscle becomes insulin resistant is unclear. We do know that there are defects in the insulin signaling cascade in NIDDM and a number of components have been shown to interfere with insulin signalling, for example intramyocellular lipids (Machann et al., 2004), serine/threonine phosphorylation of the insulin receptor proteins (Storgaard et al., 2001) and cell-surface GLUT4 content (Zierath and Wallberg-Henriksson, 2002).

We hypothesised that insulin resistance may be reflected at a transcriptional level in skeletal muscle. Knowing what genes are differentially regulated would give clues to the molecular mechanism of insulin resistance and differences in the transcription profiles of insulin resistant and insulin sensitive muscles could provide markers for the onset of diabetes. A group of young, non-obese, normoglycaemic individuals with various insulin sensitivities was recruited. The aim of the study was to generate gene expression profiles for this group using microarrays.

\section{Subjects and Methods}

Subjects. Subjects were recruited from newspaper advertisements and all subjects gave written informed consent prior to participating in this study after the procedures were explained in accordance with 
the principles of the Declaration of Helsinki as revised in 1996. The study was approved by the St. Vincent's Hospital Human Research Ethics Committee in Sydney. Healthy, sedentary, nonsmoking subjects (26 females/15 males), with no history of cardiovascular disease, dyslipidaemia, or other major health problems, were included. Exclusion criteria were age $>45 \mathrm{y}$, BMI $>32 \mathrm{~kg} / \mathrm{m}^{2}$, regular vigorous physical activity and impaired glucose tolerance. The subjects' self-assessed weight was stable ( $<2 \mathrm{~kg}$ change) for the previous 3 months.

As a screen each subject was given a euglycaemic hyperinsulinaemic clamp. The five most insulin resistant and five most insulin sensitive subjects were selected for muscle microarray analysis.

Study design. Subjects were studied over a period of approximately one month, consisting of 3 visits to our laboratory, each beginning at $8 \mathrm{am}$. Each visit was about one week apart and all procedures were performed following an overnight fast of at least 10 hours. Subjects were instructed to refrain from alcohol and exercise at least $24 \mathrm{~h}$ before testing and not to modify their dietary habits or their level of habitual physical activity throughout the assessment period. On the morning of Visit 1, anthropometric data were collected, as was a fasting blood sample. This was followed by a 75 $\mathrm{g}$ oral glucose tolerance test (OGTT) to ensure all subjects were normal glucose tolerant. On the same morning, subjects were asked to provide extensive information on their family medical history, their own medical history and on their habitual physical activity. A nutritionist recorded each subject's dietary intake (via food frequency questionnaire) during the same visit and subjects were asked to complete and return a 4-day diet diary to the laboratory about a week later. On the morning of Visit 2, a $120 \mathrm{~min}$ euglycaemic, hyperinsulinaemic clamp was performed to determine whole-body insulin sensitivity. On the third visit (Visit 3), a biopsy of the vastus lateralis muscle was performed.

Oral glucose tolerance test (OGTT). Following an overnight fast and after dietary advice was given to ensure a carbohydrate intake $>150 \mathrm{~g} /$ day over the previous 3 days, glucose tolerance was assessed by a $75 \mathrm{~g}$ OGTT. Blood samples for glucose and insulin were taken immediately before and 30, 60, 90, and $120 \mathrm{~min}$ after the glucose load. The total areas under the glucose and insulin curves (AUC) were calculated using the trapezoidal rule.

Euglycaemic, hyperinsulinaemic clamp. Subjects underwent a 120 min euglycaemic hyperinsulinaemic clamp after a $10 \mathrm{~h}$ overnight fast as previously described (DeFronzo et al., 1979; Carey et al., 1996). This dose achieved serum insulin levels of $113.2 \pm 3.9 \mathrm{mU} / \mathrm{l}$ and has previously been shown to suppress hepatic glucose production (DeFronzo et al., 1979). Intravenous cannulae were placed in each forearm, one for glucose and insulin infusions, the other retrogradely in a forearm warmed under a heating blanket for collection of arterialized plasma glucose measurements (YSI 2300; StatPlus, YellowSprings Instruments, Yellow Spring, OH). After a 30-min rest, indirect calorimetry was performed for $30 \mathrm{~min}$ and repeated during the last $30 \mathrm{~min}$ of the clamp. After basal calorimetry, insulin was infused at $50 \mathrm{mU} \cdot \mathrm{m}^{-2} \cdot \mathrm{min}^{-1}$ for $120 \mathrm{~min}$. Plasma glucose measurements were obtained every $10 \mathrm{~min}$ and the glucose infusion rate $(25 \%$ dextrose) adjusted to maintain plasma glucose levels close to $5.0 \mu \mathrm{mol} / \mathrm{l}$ (mean levels achieved were $4.97 \pm 0.04 \mu \mathrm{mol} / \mathrm{l})$. The steady-state glucose infusion rate (GIR; measured here over the final $30 \mathrm{~min}$ of the clamp) was adjusted for FFM (units $\mu \mathrm{mol} \cdot \mathrm{min}^{-1}$ $\cdot \mathrm{kg}^{-1}$ FFM) and provided an assessment of whole-body insulin sensitivity.

Skeletal muscle biopsy. Local anaesthetic (1\% lignocaine) was infused subcutaneously $\sim 20 \mathrm{~cm}$ above the patella over the vastus lateralis muscle, usually of the right leg. A percutaneous biopsy of the vastus lateralis muscle was obtained $(\sim 300 \mathrm{mg})$ according to the method of Bergstrom (1962) with a 6-mm diameter University College Hospital needle with suction assistance (Bergstrom, 1962). Upon collection, the sample was immediately blotted to remove blood, immersed in liquid nitrogen, and stored at $-80^{\circ} \mathrm{C}$ until analysed.

RNA extraction and probe preparation. RNA was extracted from muscle tissue by homogenisation in guanidinium thiocyanate followed by ultracentrifugation through a cesium chloride cushion (Chirgwin et al., 1979). RNA was quantified by absorbance at 260 $\mathrm{nm}$ and the quality assessed by absorbance ratio $\mathrm{A}_{260}: \mathrm{A}_{280}$ and denaturing gel electrophoresis (Lehrach et al., 1977). First strand cDNA was synthesised from 8-20 $\mu \mathrm{g}$ of total RNA using an oligo dT primer (Invitrogen), amino-allyl dUTP (Sigma-Aldrich) and the First-strand cDNA synthesis kit (Invitrogen). The cDNA was then purified by Sephadex G-50 chromatography and labelled with Cyanine dyes, either Cy3 or Cy5 (GE Healthcare).

Microarrays. The labelled probe was hybridised on a $19 \mathrm{~K}$ oligonucleotide array (Ramaciotti Centre, Sydney, NSW, Australia) at $51^{\circ} \mathrm{C}$ for $14-17$ hours. The array was then washed in decreasing concentrations of SSC and SDS ranging from $1 \mathrm{X}$ SSC $/ 0.2 \%$ SDS to $0.1 \mathrm{XSSC} / 0 \% \mathrm{SDS}$, and dried by centrifugation. The arrays were scanned using an Axon GenePix 4000B scanner and the resultant image analysed with GenePix Pro software (Molecular Devices Corporation, CA, USA).

As a measure of quality the arrays were normalised using the Universal ScoreCard as internal spike controls (GE Healthcare) and analysed using DiCy, an in-house database application designed to find, and cross compare, data sets based on both functionality and behaviour, as described previously (Mulligan et al., 2002; Lal et al., 2004). A statistics package written in "R" from Bioconductor (http:/ /www.bioconductor.org) was used to generate quality control plots for all the arrays.

The initial criteria for significant changes in gene expression were a $>1.1$-fold change in intensity and a threshold intensity $(>500)$, when compared to the insulin sensitive controls. As the arrays were observed to be very yellow, with minimal changes, a low fold change was used to avoid omitting important genes. Each spot was also required to be of good quality, as assigned by a flag of zero in the GenePix program.

Real time RT-PCR. Total RNA from muscle biopsies was treated with RQ1 RNase-free DNase (Promega) and $0.05 \mu \mathrm{g}$ was then reverse transcribed using the First-strand cDNA synthesis kit (Invitrogen) with a random hexamer primer. The resulting cDNA was diluted $1: 8$ and $1: 64$. For each gene investigated the cDNA from each subject was tested in duplicate at the original 


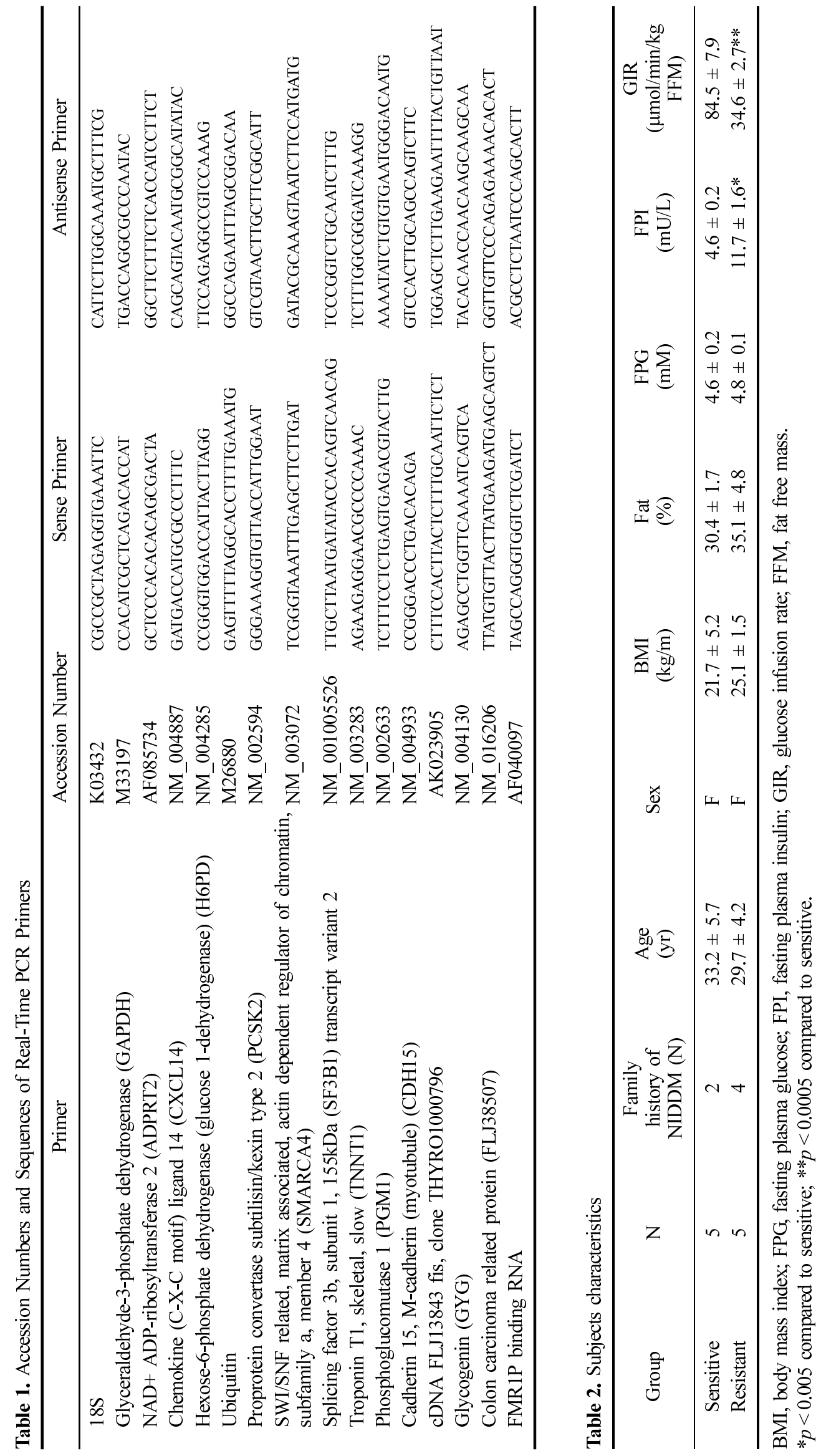


concentration and for each of the dilutions. Primer concentration used for each gene was $300 \mathrm{nM}$. The abundance of $18 \mathrm{~S}$ was used as an internal control for the amount of cDNA in a sample, since expression levels of $18 \mathrm{~S}$ are assumed to remain unchanged between samples.

\section{Results}

Clinical characteristics of the subjects. The mean fasting plasma glucose concentrations were similar for the insulin resistant and insulin sensitive groups and their OGTT responses were not significantly different. The two groups were matched for age, sex, BMI and \% fat, as shown in Table 2. The mean fasting plasma insulin concentration was $4.6 \pm 0.2 \mathrm{mU} / \mathrm{l}$ in the 5 insulin sensitive subjects and $11.7 \pm 1.6 \mathrm{mU} / 1$ in the 5 insulin resistant subjects $(p<0.005)$. The average glucose infusion rate during the hyperinsulinaemic euglycaemic clamp was $84.5 \pm 7.9 \mu \mathrm{mol} \cdot \mathrm{min}^{-1} \cdot \mathrm{kg}^{-1}$ fat free mass for insulin sensitive subjects and $34.6 \pm 2.7 \mu \mathrm{mol} / \mathrm{min} / \mathrm{kg}$ fat free mass for insulin resistant subjects $(p<0.0005)$. Four of five insulin resistant subjects were first degree relatives of patients with type 2 diabetes, while only two out of five insulin sensitive subjects were relatives of type 2 diabetics. This highlights the fact that while generally relatives of diabetic patients are insulin resistant, the degree of insulin resistance covers a broad spectrum, despite family history.

Quality control. The R package from Bioconductor confirmed that the arrays were of good quality as shown in the boxplot in Fig. 1. The majority of genes are concentrated around the "no change" mark. Most genes should not change in any microarray study. The MA plot in Fig. 2 is

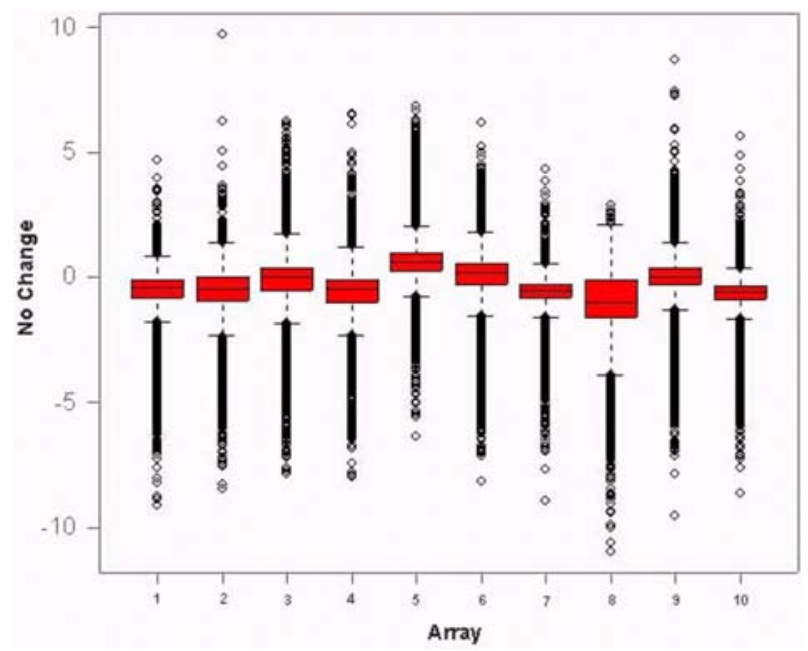

Fig. 1. Boxplots of all arrays. The central red boxes represent the inter-quartile range (IQR), which is defined as the difference between the 75th percentile and 25th percentile. Extreme values are plotted individually as seen by the block dots above and below the central box. representative of all arrays and shows a spread of all spots around the centre line. Spots around the centre line are predominantly yellow and do not exhibit a change in gene expression. The symmetry of this plot also confirms that the arrays were of good quality. GenePix "flags" each spot based on its quality and good quality spots, i.e. Flag $=0$, were selected.

Microarray analysis. The transcriptome appears to be very stable and the only way we could find genes to study further was to relax the criteria fold change to 1.1 and investigate a gene further if it displayed a change in 7 or 8 of 10 arrays. The low fold change created large, loose criteria based sets. These sets were then combined to identify common, similarly expressed genes in the insulin resistant and sensitive state (Table 3-4).

However, it is important to note that despite these very loose criteria based sets, the numbers of differentially expressed genes was only in the low thousands out of a total of 19,000 genes on each array. When these sets were combined less than 20 genes were common between arrays.

Real-time PCR. Quantitative Real-Time PCR was performed on 10 up-regulated genes and 4 down-regulated genes to confirm microarray data.

Very small fold changes were observed once $\mathrm{C}_{\mathrm{T}}$ values had been normalised to the internal control of $18 \mathrm{~S}$ (Table 5). Glyceraldehyde dehydrogenase (GAPdh), often used as a housekeeping gene, was also measured in each sample and showed strong and constant gene expression levels across all samples.

Average fold changes observed in real-time PCR were consistent with fold changes observed on microarrays.

\section{Discussion}

We hypothesised that changes in gene expression may be an early indicator of the metabolic syndrome, but our study demonstrated that changes in gene expression are not detectable in skeletal muscle in insulin resistance. Gene expression profiles of 19,000 genes were relatively similar in

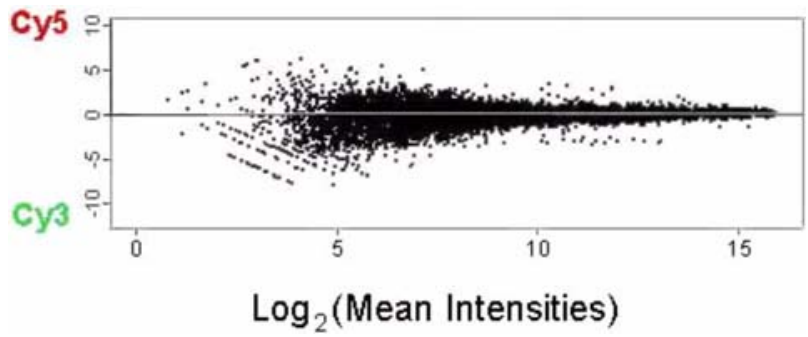

Fig. 2. MA-plot showing the relationship between "M" (Cy5/ $\mathrm{Cy} 3$ ratios) on the $\mathrm{y}$-axis and "A", the distribution of average intensities on the $\mathrm{x}$-axis. 
Table 3. Upregulated genes in insulin resistance in 8 out of 10 arrays

\begin{tabular}{ll}
\hline Accession & \multicolumn{1}{c}{ Gene Name } \\
\hline NM_006193 & Homo sapiens paired box gene 4 (PAX4) mRNA \\
NM_002594 & Homo sapiens proprotein convertase subtilisin/kexin type 2 (PCSK2) mRNA \\
NM_004933 & Homo sapiens cadherin 15, M-cadherin (myotubule) (CDH15) mRNA \\
NM_012433 & Homo sapiens splicing factor 3b, subunit 1, 155kD (SF3B1) mRNA \\
AK001299 & Homo sapiens cDNA FLJ10437 fis, clone NT2RP1000581 \\
NM_003283 & Homo sapiens troponin T1, skeletal, slow (TNNT1) mRNA \\
NM_004285 & Homo sapiens hexose-6-phosphate dehydrogenase (glucose 1-dehydrogenase) (H6PD) mRNA \\
NM_004887 & Homo sapiens CXC chemokine in breast and kidney (BRAK) mRNA \\
J04948 & Human alkaline phosphatase (ALP-1) mRNA \\
M26880 & Human ubiquitin mRNA \\
AF085734 & Homo sapiens NAD+ ADP-ribosyltransferase 2 (ADPRT2) mRNA \\
NM_002594 & Homo sapiens proprotein convertase subtilisin/kexin type 2 (PCSK2) mRNA \\
NM_002633 & Homo sapiens phosphoglucomutase 1 (PGM1) mRNA \\
NM_002476 & Homo sapiens myosin, light polypeptide 4, alkali; atrial, embryonic (MYL4), mRNA \\
M31212 & Human myosin light chain 3 non-muscle (MLC3nm) mRNA, complete cds \\
NM_000440 & Homo sapiens phosphodiesterase 6A, cGMP-specific, rod, alpha (PDE6A), mRNA
\end{tabular}

Table 4. Downregulated genes in insulin resistance in 7 out of 10 arrays

\begin{tabular}{ll}
\hline Accession & \multicolumn{1}{c}{ Gene Name } \\
\hline NM_016206 & Homo sapiens colon carcinoma related protein (LOC51159), mRNA \\
NM_004130 & Homo sapiens glycogenin (GYG) mRNA \\
AK023905 & Homo sapiens cDNA FLJ13843 fis, clone THYRO1000796 \\
AF040097 & Homo sapiens FMR1P binding RNA, partial sequence (1p36.13-36.23) \\
NM_006450 & Homo sapiens splicing factor (45kD) (SPF45) mRNA \\
NM_006540 & Homo sapiens nuclear receptor coactivator 2 (NCOA2), mRNA \\
NM_003072 & Homo sapiens SWI/SNF related, matrix associated, actin dependent regulator of chromatin, \\
& subfamily a, member 4 (SMARCA4) mRNA \\
\hline
\end{tabular}

the insulin resistant and insulin sensitive states. We did observe some insignificant fold increases but no changes greater than 3 fold were consistently observed across the arrays.

Obviously, there may be genes of interest not present on the array, which do display a change in gene expression in skeletal muscle in insulin resistance. We confirmed that the arrays were of good quality and believed that the selected genes warranted further investigation. The microarray expression levels for 14 selected genes were successfully confirmed with real time PCR (Table 5 and Fig. 3).

We were expecting a difference in the gene expression profiles as the insulin sensitivities of our subject groups were very different. The insulin resistant subjects exhibited a 2-fold lower insulin sensitivity (glucose infusion rate and fasting plasma insulin) compared to the insulin sensitive subjects. The subject group was also young, non-obese and normoglycaemic, which removes some confounding factors present in other human studies. This suggests that insulin resistance is not due to transcriptional changes in skeletal muscle, the primary organ responsible for glucose uptake. We do know that a defect is present in parts of the insulin signalling pathway of skeletal muscle in insulin resistance, as evidenced by several studies (Cusi et al., 2000; Brozinick et al., 2003; Hojlund et al., 2003). However, gene expression does not appear to be the cause of this defect. In fact, gene expression of skeletal muscle in insulin resistance is much the same as in insulin sensitive individuals.

There have been numerous studies using microarray technology to investigate gene expression in different metabolic states, as summarized by Permana et al. (2004) (Permana et al., 2004). Some of these studies focused on gene expression in insulin resistance and type 2 diabetes. For example, Sreekumar et al. (2002) investigated the effect of insulin treatment on skeletal muscle in type 2 diabetics and found several genes with altered expression. Although the type 2 diabetic subjects were insulin resistant, they were also hyperglycaemic (Sreekumar et al., 2002). This factor sets them apart from our normoglycaemic subject group.

Another study by Yang et al. (2002) compared gene 
Table 1. Quantitative Real-time PCR normalised $C_{T}$ and fold change values for the selected genes in Table 1. SYBR ${ }^{\circledR}$ Green dye was used for product detection

\begin{tabular}{lccc}
\hline \multicolumn{1}{c}{ Gene } & $\begin{array}{c}\text { Average } \\
\text { fold } \\
\text { decrease in } \\
\text { insulin } \\
\text { resistance }\end{array}$ & $\begin{array}{c}\text { Average } \\
\text { fold } \\
\text { insulin } \\
\text { resistance }\end{array}$ & \\
& & 1.11 & 0.53 \\
\hline GAPdh & & 1.19 & 0.52 \\
ADPRT2 & & 1.40 & 0.33 \\
CXC chemokine & 1.07 & & 0.82 \\
H6Pdh & & 1.67 & 0.22 \\
Ubiquitin & 1.03 & & 0.88 \\
Glycogenin & & 1.53 & 0.47 \\
PCSK2 & 1.12 & & 0.68 \\
SWI/SNF related & & 1.18 & 0.48 \\
Splicing Factor 3b & & 1.23 & 0.46 \\
FMR1P & 1.09 & & 0.72 \\
Troponin & & 1.34 & 0.37 \\
Phosphoglucomutase & & 1.18 & 0.37 \\
Cadherin & 1.00 & 1.00 & 0.99 \\
Colon carcinoma related & & 1.17 & 0.64 \\
FLJ13843 fis, clone & & &
\end{tabular}

expression in insulin resistant obese subjects (Yang et al., 2002). A large number of transcripts were found to be differentially expressed (185), but only $20 \%$ were true positives. However, the obesity of the subjects may have been a confounding factor. These two studies by Sreekumar et al. (2002) and Yang et al. (2002), did not investigate the gene expression in insulin resistance alone.

Other studies have compared the expression of individual or small groups of genes in skeletal muscle and have found insignificant changes in gene expression in insulin resistance and type 2 diabetes.

A study by Thompson et al. (1996) (Thompson et al., 1996) measured a number of different transcripts in insulin resistant and insulin sensitive human skeletal muscle before, during and after a 2 hour insulin infusion. At basal level there was no difference in expression of the insulin receptor, GLUT4, PPP1A, c-fos and c-jun. Also, over the $2 \mathrm{~h}$ insulin infusion, the levels of c-fos and GLUT4 did not change significantly between the two groups. Furthermore, the degree of obesity was significantly different between study groups.

Our study measured gene expression at a basal level and found that a large number of genes may not be different. Despite higher fasting plasma insulin levels, the level of expression was equivocal. It is possible that we would have seen some changes with glucose or insulin administration.

Bao et al. (1998) found no difference in the expression of $\mathrm{UCP} 2, \mathrm{UCP} 3 \mathrm{~S}$ and UCP3L between insulin sensitive and insulin resistant subjects (Bao et al., 1998). It was intriguing that hyperglycaemic type 2 diabetic subjects and normoglycaemic

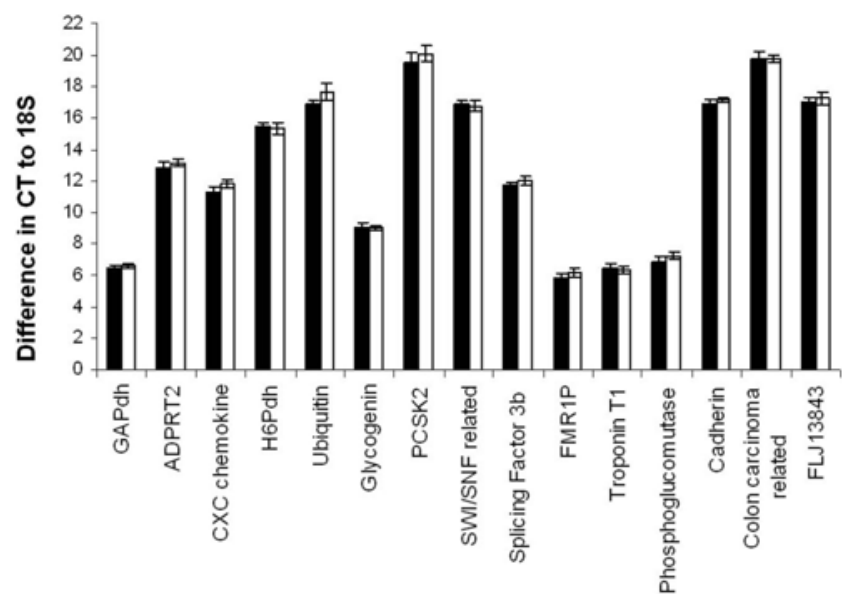

Fig. 3. Average $C_{T}$ values for 15 genes confirmed using real time PCR when compared to the expression of $18 \mathrm{~S}$, where black represents values for insulin resistant subjects and white represents values for insulin sensitive subjects. $\mathbf{\square}$ : Insulin resistant subjects, $\square$ : Insulin sensitive subjects.

obese subjects showed increased expression of UCPs. Bao et al. (1998) therefore concluded that a factor other than increased fasting insulin and decreased glucose uptake rates, increased the expression of UCPs in muscle (Bao et al., 1998). Insulin resistance alone was therefore not implicated in producing changes in gene expression. Therefore, transcription is only seen when obesity and/or hyperglycaemia are present.

Most studies focus on changes in gene expression in disease. Only a small number of studies have shown no changes in individual or small groups of genes in insulin resistance. It is difficult to compare our results to these studies as they often involve subjects that are obese or type 2 diabetics.

The major strength of our study was the use of two young, non-obese, normoglycaemic subject groups, differing only in their insulin sensitivity. Therefore, major confounding factors were removed, presenting a clear comparison, where insulin resistance was the sole metabolic condition. We can therefore conclude that skeletal muscle gene expression (in 19,000 genes) is not a cause for insulin resistance and cannot be used as an early indicator of the syndrome. Also, if a permanent gene expression defect is not present the resistance to insulin may be more likely to be reversible.

\section{Conclusions}

Our data show that insulin resistance is not reflected in the transcription profile of skeletal muscle. This demonstrates that transcriptional changes do not contribute to the disease and that skeletal muscle does not display changes in gene expression during insulin resistance. 


\section{References}

Bao, S., Kennedy, A., Wojciechowski, B., Wallace, P., Ganaway, E. and Garvey, W. T. (1998) Expression of mRNAs encoding uncoupling proteins in human skeletal muscle: effects of obesity and diabetes. Diabetes 47, 1935-1940.

Bergstrom, J. (1962) Muscle electrolytes in man. Scand. J. Clin. Lab. Investi. 14, 1-100.

Brozinick, J. T., Jr., Roberts, B. R. and Dohm, G. L. (2003) Defective signaling through Akt-2 and -3 but not Akt-1 in insulin-resistant human skeletal muscle: potential role in insulin resistance. Diabetes 52, 935-941.

Carey, D. G., Jenkins, A. B., Campbell, L. V., Freund, J. and Chisholm, D. J. (1996) Abdominal fat and insulin resistance in normal and overweight women: Direct measurements reveal a strong relationship in subjects at both low and high risk of NIDDM. Diabetes 45, 633-638.

Chirgwin, J. M., Przybyla, A. E., MacDonald, R. J. and Rutter, W. J. (1979) Isolation of biologically active ribonucleic acid from sources enriched in ribonuclease. Biochemistry 18, 5294-5299.

Cusi, K., Maezono, K., Osman, A., Pendergrass, M., Patti, M. E., Pratipanawatr, T., DeFronzo, R. A., Kahn, C. R. and Mandarino, L. J. (2000) Insulin resistance differentially affects the PI 3-kinase- and MAP kinase-mediated signaling in human muscle. J. Clin. Invest. 105, 311-320.

Dandona, P., Aljada, A. and Bandyopadhyay, A. (2004) Inflammation: the link between insulin resistance, obesity and diabetes. Trends Immunol. 25, 4-7.

DeFronzo, R. A., Tobin, J. D. and Andres, R. (1979) Glucose clamp technique: a method for quantifying insulin secretion and resistance. Am. J. Physiol. 237, 214-223.

Felber, J. P. and Golay, A. (2002) Pathways from obesity to diabetes. Int. J. Obes. Relat. Metab. Disord. 26, 39-45.

Goldstein, B. J. (2002) Insulin resistance as the core defect in type 2 diabetes mellitus. Am. J. Cardiol. 90, 3-10.

Goldstein, B. J. (2003) Insulin resistance: from benign to type 2 diabetes mellitus. Rev. Cardiovasc. Med. 4, 3-10.

Haffner, S. M. (2003a) Insulin resistance, inflammation, and the prediabetic state. Am. J. Cardiol. 92, 18-26.

Haffner, S. M. (2003b) Pre-diabetes, insulin resistance, inflammation and CVD risk. Diabetes Res. Clin. Pract. 61, 9-18.

Hojlund, K., Staehr, P., Hansen, B. F., Green, K. A., Hardie, D. G., Richter, E. A., Beck-Nielsen, H. and Wojtaszewski, J. F. (2003) Increased phosphorylation of skeletal muscle glycogen synthase at NH2-terminal sites during physiological hyperinsulinemia in type 2 diabetes. Diabetes 52, 1393-1402.

Kahn, B. B. and Flier, J. S. (2000) Obesity and insulin resistance. J. Clin. Invest. 106, 473-481.

Kopelman, P. G. (2000) Obesity as a medical problem. Nature 404, 635-643.

Lal, S., Lui, R., Nguyen, L., Macdonald, P., Denyer, G. and dos
Remedios, C. (2004) Increases in leukocyte cluster of differentiation antigen expression during cardiopulmonary bypass in patients undergoing heart transplantation. Proteomics 4, 1918-1926.

Lehrach, H., Diamond, D., Wozney, J. M. and Boedtker, H. (1977) RNA molecular weight determinations by gel electrophoresis under denaturing conditions, a critical reexamination. Biochemistry 16, 4743-4751.

Machann, J., Haring, H., Schick, F. and Stumvoll, M. (2004) Intramyocellular lipids and insulin resistance. Diabetes Obes. Metab. 6, 239-248.

Mulligan, C., Rochford, J., Denyer, G., Stephens, R., Yeo, G., Freeman, T., Siddle, K. and O'Rahilly, S. (2002) Microarray analysis of insulin and insulin-like growth factor-1 (IGF-1) receptor signaling reveals the selective up-regulation of the mitogen heparin-binding EGF-like growth factor by IGF-1. $J$. Biol. Chem. 277, 42480-42487.

Permana, P. A., Del Parigi, A. and Tataranni, P. A. (2004) Microarray gene expression profiling in obesity and insulin resistance. Nutrition 20, 134-138.

Petersen, K. F. and Shulman, G. I. (2002) Pathogenesis of skeletal muscle insulin resistance in type 2 diabetes mellitus. Am. J. Cardiol. 90, 11-18.

Smith, U. (2002) Impaired ('diabetic') insulin signaling and action occur in fat cells long before glucose intolerance--is insulin resistance initiated in the adipose tissue? Int. J. Obes. Relat. Metab. Disord 26, 897-904.

Sowers, J. R. (2004) Insulin resistance and hypertension. Am. J. Physiol. Heart Circ. Physiol. 286, 1597-1602.

Sreekumar, R., Halvatsiotis, P., Schimke, J. C. and Nair, K. S. (2002) Gene expression profile in skeletal muscle of type 2 diabetes and the effect of insulin treatment. Diabetes 51, 19131920.

Storgaard, H., Song, X. M., Jensen, C. B., Madsbad, S., Bjornholm, M., Vaag, A. and Zierath, J. R. (2001) Insulin signal transduction in skeletal muscle from glucose-intolerant relatives of type 2 diabetic patients. Diabetes 50, 2770-2778.

Thompson, D. B., de Gregorio, M. and Sommercorn, J. (1996) Insulin regulation of multiple ribonucleic acid species in human skeletal muscle in insulin-sensitive and insulin-resistant subjects. J. Clin. Endocrinol. Metab. 81, 519-523.

Yang, X., Pratley, R. E., Tokraks, S., Bogardus, C. and Permana, P. A. (2002) Microarray profiling of skeletal muscle tissues from equally obese, non-diabetic insulin-sensitive and insulinresistant Pima Indians. Diabetologia 45, 1584-1593.

Yki-Jarvinen, H. (1995) Role of insulin resistance in the pathogenesis of NIDDM. Diabetologia 38, 1378-1388.

Zierath, J. R. and Wallberg-Henriksson, H. (2002) From receptor to effector: insulin signal transduction in skeletal muscle from type II diabetic patients. Ann. N. Y. Acad. Sci. 967, 120-134. 